\title{
Flow Patterns, Void Fraction, Pressure Drop, and Convective Heat Transfer in Gas-Liquid Two Phase Flow in Various Pipe Inclinations
}

\author{
Afshin J. Ghajar \\ Regents Professor and John Brammer Endowed Professor School of Mechanical and Aerospace Engineering \\ Oklahoma State University \\ Stillwater, Oklahoma, USA \\ afshin.ghajar@okstate.edu
}

The phenomenon of gas-liquid two-phase flow in inclined systems, although not as common as horizontal or vertical flow, is of great practical significance in several applications such as undulating oil-gas flow lines, chemical process engineering, inclined flow paths in steam condensers and generators. In these practical applications, accurate determination of two-phase flow variables such as void fraction, pressure drop and heat transfer is of great importance for system sizing and optimization. It is well established fact that these parameters are very sensitive to the spatial and morphological variations of the two-phase flow structure. The two-phase flow structure commonly termed as flow pattern, depends on the interaction and balance between the buoyancy-inertia-gravity forces which in turn are a function of pipe inclination. Thus, a correct understanding of the effect of change in pipe inclination on the two-phase flow structure is needed and its effect on the thermofluidics of two-phase flow needs to be understood. Thus, the scope of this lecture is to present an overview of the pipe inclination effects of the gas-liquid two-phase flow phenomenon.

The pipe inclination effects on non-boiling two-phase flow are studied through the data available in literature and extensive experiments carried out in Two Phase Heat Transfer Laboratory at Oklahoma State University. These experiments are carried out in two different test rigs of $0.5 \mathrm{inch}$ and 1 inch I.D. respectively, using air-water as fluid combination. These experimental setups are unique as they can be inclined to in both upward and downward inclinations and are capable of flow visualization and simultaneous measurements of void fraction, pressure drop, and heat transfer. The experimental data show the flow pattern and pipe inclination dependency of all two-phase flow variables. At lower mass fluxes, this effect is found to be most significant where the two-phase flow phenomenon is dominated by the buoyant forces acting on the gas phase. Experiments also reveal the prevalence and insights about the flow reversal and transient nature of the two-phase flow in upward and downward inclined systems.

Two-phase flow literature reports a plethora of correlations/models for determination of void fraction, pressure drop and non-boiling heat transfer. Since the two-phase flow is a function of several variables such as flow patterns, fluid properties, and pipe diameter and inclination, it is quite a challenging task for the end user to select an appropriate flow condition specific correlation/model. Selection of a correct model also requires some fundamental understanding of the two-phase flow physics and the underlying principles/ assumptions/limitations associated with these correlations. To address these issues, this lecture also introduces some of the top performing two-phase flow models validated against a comprehensive data set. 